\title{
Phenylene ring dynamics in phenoxy and the effect of intramolecular linkages on the dynamics of some engineering thermoplastics below the glass transition temperature
}

\author{
Silvia Arrese-Igor, ${ }^{1}$ Arantxa Arbe, ${ }^{1}$ Angel Alegría,,${ }^{1,2}$ Juan Colmenero, ${ }^{1,2,3}$ and Bernhard Frick ${ }^{4}$ \\ ${ }^{1}$ Centro Física de Materiales (CSIC-UPV/EHU), Apartado 1072, 20080 San Sebastián, Spain \\ ${ }^{2}$ Departamento de Física de Materiales, UPV/EHU, Apartado 1072, 20080 San Sebastián, Spain \\ ${ }^{3}$ Donostia International Physics Center, Apartado 1072, 20080 San Sebastián, Spain \\ ${ }^{4}$ Institut Laue-Langevin, Boîte Postale 156, 38042 Grenoble Cedex 9, France
}

(Received 28 August 2006; published 4 May 2007)

\begin{abstract}
We have investigated the dynamics of phenylene rings in the engineering thermoplastic bisphenol-A poly(hydroxyether) - phenoxy-below its glass transition temperature by means of neutron scattering techniques. A relatively wide dynamic range has been covered thanks to the combination of two different types of neutron spectrometers, time of flight and backscattering. Partially deuterated samples have been used in order to isolate the phenylene ring dynamics. The resulting neutron scattering signal of phenoxy has been described by a model that considers $\pi$ flips and oscillation motions for phenylene rings. The associated time scales are broadly distributed with mean activation energies equal to 0.41 and $0.21 \mathrm{eV}$, respectively. Finally, a comparative study with the literature shows that the dielectric and mechanical $\gamma$ relaxation in phenoxy exhibit good correlation with the characteristic times of the aliphatic chain published elsewhere and with the characteristic times observed for the motion of phenylene rings by neutron scattering. These findings are discussed in a more general framework that considers, in addition, previous results on other polymers, which also contain the bisphenol-A unit.
\end{abstract}

DOI: 10.1103/PhysRevE.75.051801

PACS number(s): 61.41.+e, 61.12.Ex, 83.10.Mj

\section{INTRODUCTION}

Engineering thermoplastics based on the bisphenol-A moiety (BPA) have an important technological significance. Their excellent mechanical and thermal properties make them suitable for a huge number of applications. Although the microscopic origin of these interesting mechanical properties is not completely elucidated yet, it has been long believed that they are closely related with the so-called secondary relaxation processes, i.e., with those dynamic processes observed by spectroscopic conventional techniques such as dielectric (DS) or mechanical (MS) spectroscopy. These techniques allow a good characterization of the shape and characteristic frequency of secondary relaxations in a broad frequency range $\left(\sim 10^{-3}-\sim 10^{9} \mathrm{~Hz}\right.$ with standard techniques) $[1,2]$. However, such measurements do not provide microscopic spatial information of the molecular motions involved. As a consequence, some technique allowing the characterization of the motions at a molecular level is needed in order to identify the molecular origin of the secondary relaxations. To the present date most of the advances in the field were obtained by means of nuclear magnetic resonance (NMR) [3-8]. In recent years, however, we have also been approaching the problem from a different point of view using neutron scattering (NS) to characterize the molecular motions below the glass transition temperature $T_{g}$ [9-17]. NS techniques perfectly suit this task as they offer the possibility of selective labeling through deuteration, and provide spatial information at a molecular level. A deep knowledge of the mechanisms and specific nature of the molecular motions behind the secondary relaxations will remarkably contribute to the understanding of the structure/dynamics/properties relationship, which would ultimately enable tailoring the polymer properties for demanding applications.
In this work, we have focused on a widely used polymer also containing the BPA unit, poly(hydroxyether)-phenoxy, $\mathrm{PH}-$. The range of applications of $\mathrm{PH}$ comprises, e.g., adhesives, coatings, plastics, or composites. The linking group between the BPA moieties is an aliphatic chain (see Fig. 1). Our previous NS studies on other thermoplastics have evidenced the very close relation between the BPA phenylene rings and the secondary relaxations $[13,14,18]$. For this reason, here we have investigated the sub- $T_{g}$ phenylene ring dynamics of phenoxy in different time scales combining time of flight $\left(\mathrm{TOF}, \simeq 3 \times 10^{-13}-3 \times 10^{-11} \mathrm{~s}\right.$ ) and backscattering $\left(\mathrm{BS}, \simeq 10^{-10}-10^{-9} \mathrm{~s}\right) \mathrm{NS}$ techniques. The selectivity of NS when combined with isotopic substitution has been exploited using selective protonation for phenylene rings. In this way, the scattered intensity mainly reveals the dynamics of these groups through the incoherent scattering function of hydrogen. To account for the coherent contributions to the scattered intensity we have also performed diffraction measure-

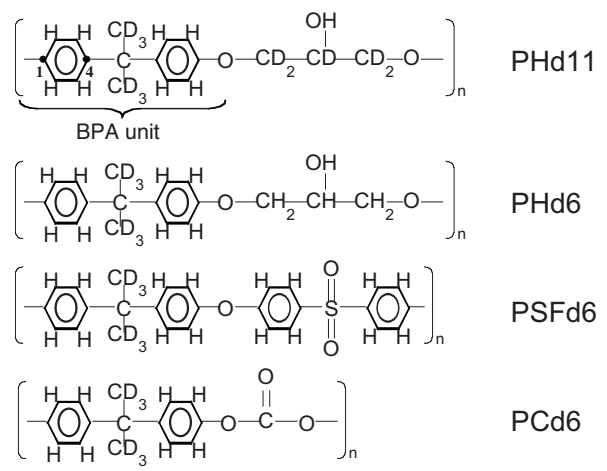

FIG. 1. Molecular formula for PH, PSF, and PC with deuterated methyl groups (d6), and $\mathrm{PH}$ with both methyl groups and aliphatic chain deuterated (d11). 
ments with polarization analysis, which in addition provide information on the short range order of this system.

In the first part of this paper new results concerning the diffraction experiments and the phenylene ring dynamics in PH by NS will be exposed. Next, a summary of phenylene ring dynamics in $\mathrm{PH}$ together with previous results for polysulfone (PSF) and polycarbonate (PC), which also contain the BPA unit, will be presented. Finally, we discuss the connection between phenylene ring dynamics and the secondary relaxations observed by spectroscopic techniques in these polymers.

\section{EXPERIMENTAL}

\section{A. Samples}

In Fig. 1 the chemical formula of the repeating units of PH samples investigated in this work, with methyl groups and aliphatic chain deuterated (d11), and with only methyl groups deuterated (d6) are shown. Molecular weight and polydispersity of the samples supplied by Polymer Source were $M_{n}=20.9 \mathrm{~kg} / \mathrm{mol}$ and $M_{n}=7.7 \mathrm{~kg} / \mathrm{mol}$, and $M_{w} / M_{n}$ $=2.76$ and $M_{w} / M_{n}=1.76$ for PHd11 and PHd6, respectively. Differential scanning calorimetry measurements lead to $T_{g}$ values for both samples around $360 \mathrm{~K}$. We note that samples were vacuum heated near $T_{g}$ for 48 hours prior to the NS experiments.

\section{B. NS experiments}

The quantity assessed in a neutron scattering experiment is the double differential cross section $\frac{\partial^{2} \sigma}{\partial \Omega \partial E}$ : the number of neutrons scattered into a solid angle between $\Omega$ and $\Omega+d \Omega$, after having exchanged an energy between $E$ and $E+d E$ with the sample, relative to the number of incident neutrons per unit area. The double scattering cross section can be separated in a sum of incoherent and coherent contributions coming from the scattering of different nuclei within the sample, and having the information of the individual and collective motion and arrangement of the atoms, respectively [19]. Each of these contributions is weighted by the incoherent $\left(\sigma_{\text {inc }}\right)$ and coherent $\left(\sigma_{c o h}\right)$ scattering cross sections of the atoms. The incoherent scattering cross section of hydrogen is much bigger than the cross sections of the rest of the typical nuclei within a polymeric sample, therefore, generally the incoherent scattering from hydrogen dominates the measured intensity in samples that contain it. We note that incoherent contributions coming from atoms other than hydrogen amount only to at most $3 \%$ of the signal, which can be safely neglected within the accuracy of the data.

\section{Diffraction with polarization analysis}

Experimentally, it is possible to separate the coherent and incoherent contributions to the scattering by using a spinpolarized neutron beam and polarization analysis [19]. The experiments with polarization analysis reported here were carried out by means of the D7 spectrometer at the Institute Laue Langevin (ILL, Grenoble, France). Measurements were conducted at 10 and $300 \mathrm{~K}$ without energy analysis for structural characterization and quantification of the relative con-
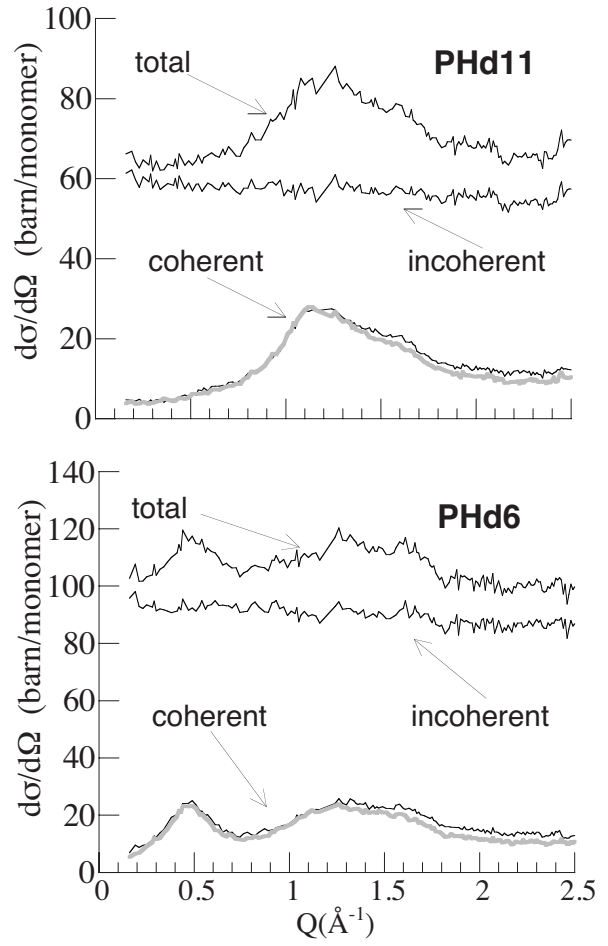

FIG. 2. Total $(10 \mathrm{~K})$, incoherent $(10 \mathrm{~K})$, and coherent (black and gray lines at 10 and $300 \mathrm{~K}$, respectively) differential cross sections measured for PHd11 and PHd6 by D7 at ILL. The error is less than $3 \%$ for all the lines shown.

tribution of the coherent and incoherent components to the total intensity. The wavelength used was $4.84 \AA$, allowing us to cover a $Q$ range between 0.16 and $2.51 \AA^{-1}$.

\section{Quasielastic neutron scattering}

We have investigated the dynamics of phenylene rings in glassy $\mathrm{PH}$ by means of quasielastic neutron scattering. As in our previous works on PC and PSF [13-16] two different types of neutron spectrometers, TOF and BS, were used in order to cover a wide dynamic range that extends from microscopic $\left(\sim 10^{-13} \mathrm{~s}\right)$ to mesoscopic $\left(\sim 10^{-9} \mathrm{~s}\right)$ times. TOF measurements (microscopic time scale) were conducted on the PHd6 sample in the instrument IN6 at the ILL. BS measurements (mesoscopic time scale) were performed on both $\mathrm{PH}$ samples in the instrument IN16 also at the ILL. In all the cases spectra were recorded at $10 \mathrm{~K}$ and from $50 \mathrm{~K}$ up to $T_{g}$ every $50 \mathrm{~K}$. The transmission of the samples was close to $\sim 90 \%$ in order to achieve a compromise between reasonable statistics and negligible multiple scattering effects. The study of PH was completely analogous to those performed earlier on PSF and PC. Therefore, further details on the experiments can be found in Refs. [13-16].

\section{RESULTS}

\section{A. D7 measurements: Structure}

Figure 2 shows the results of the diffraction experiments. The partial structure factors revealing the structural information reflect the different pair correlations dominating the co- 
herent intensity for each sample. Partial structure factors (coherent intensity) present different maxima and shoulder at around $0.5,1.25$, and $1.6 \AA^{-1}$. Due to the different deuteration of the samples, the relative weights of the pair correlation functions involving these isotopes are different in PHd11 and PHd6 and so are the intensities of the mentioned shoulder and maxima. As can be deduced from other studies of the short range order in polymers [20,21], the assignation of a specific correlation to the observed peaks is uncertain in systems with complex microstructure like $\mathrm{PH}$. Based on the $T$-dependence of the maximum position, the peaks in the region between 1 and $1.5 \AA^{-1}$ are usually ascribed to have a predominantly intermolecular character (see, e.g., Refs. $[22,23])$. Therefore, the peak at $\sim 1.25 \AA^{-1}, Q / 2 \pi \sim 5 \AA$ may probably reflect interchain correlations. In this case, as measurements have been performed in the glassy state, effects of thermal expansion might be negligible to lead to an appreciable shift of the main peak with $T$. We note that this peak appears in all the samples investigated here and also in the previous works on PC and PSF $[13,14]$. Unfortunately, the precise characterization of the local structural properties in these kind of systems is very complicated and demands the combination of diffraction experiments and MD simulations, which is beyond the scope of this work.

On the other hand, as expected from the ratio between incoherent and total scattering cross sections $\left(\frac{\sigma_{i n c}}{\sigma_{t o t}}=0.86\right.$ for PHd6 and 0.79 for PHd11) the incoherent scattering dominates the total scattered intensity in both samples. In the case of PHd6 the $Q$ modulation of the total intensity coming from the coherent contribution is weak, whereas it is more noticeable in PHd11. These results will be used later for the analysis and characterization of the NS measurements with energy or time resolution.

\section{B. Dynamics}

\section{TOF measurements: The microscopic time scale}

The experimental scattering functions $I_{\text {expt }}(Q, \omega)$ obtained by means of IN6 in the microscopic time scale for PHd6 show increasing quasielastic (see Fig. 3) and decreasing elastic intensity with temperature. The good statistics of the data (error less than 3\%) allows one to clearly distinguish the boson peak at energies $\sim 2 \mathrm{meV}$. With increasing temperature, a quasielastic contribution in $I_{\text {expt }}(Q, \omega)$ fills up the region between the boson peak and the elastic line. This quasielastic broadening can be produced by the onset of anharmonic vibrations, by the so-called "fast dynamics," and/or by some molecular motion related to relaxation processes. From the decrease of the elastic intensity with increasing $Q$ a mean square displacement $\left[\left\langle u^{2}\right\rangle(T)\right]$ can be defined at each temperature by $I_{\max }(Q, T) / I_{\max }(Q, T=10 \mathrm{~K})$ $=P(T) \exp \left[-\left\langle u^{2}\right\rangle(T) Q^{2} / 3\right]$. The so obtained mean square displacement, which may depend on the resolution, is plotted in the inset of Fig. 3 together with those previously obtained under analogous conditions and in the same way for PSF and PC. We note that the results on $\left\langle u^{2}\right\rangle(T)$ from IN6 data are indistinguishable if instead of the maximum value of the scattered intensity $I_{\max }(Q, T)$ the integral over \pm HWHM is

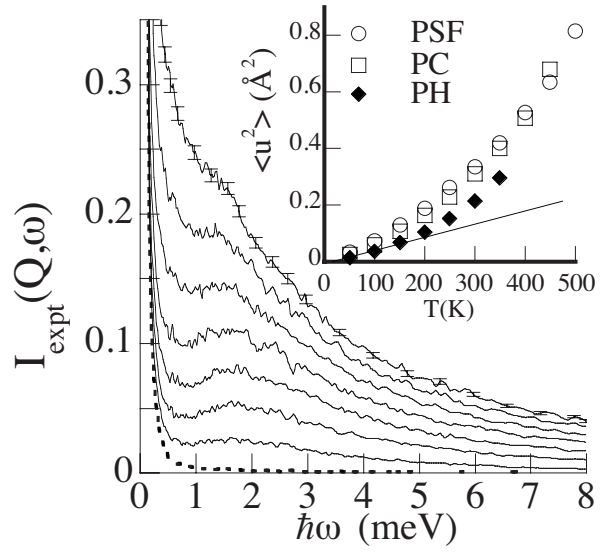

FIG. 3. Experimental scattering functions $I_{\text {expt }}(Q, \omega)$ of PHd6 in the microscopic time scale at $Q=1.94 \AA^{-1}$ and at temperatures from 50 (bottom) to $350 \mathrm{~K}$ (top) every $50 \mathrm{~K}$. For clearness only error bars for the $350 \mathrm{~K}$ spectrum have been depicted but they are representative of the error at all temperatures. The $10 \mathrm{~K}$ spectrum is shown by the dotted line. Inset: mean square displacement obtained from TOF experiments in PSF, PC, and PHd6. The size of the symbols is indicative of the error. The line represents the expected harmonic $T$ dependence for $\mathrm{PH}$.

considered. On the other hand, values close to 1 have been found for the prefactor $P(T)$ in all cases.

In order to uncouple phenylene ring motions from other "fast dynamic processes" taking place at times shorter than $\sim 1-2 \mathrm{ps}$, the $I_{\text {expt }}(Q, \omega)$ spectra were Fourier transformed to the time domain. Figure 4 shows that for PHd6 the intermediate scattering functions obtained from the Fourier transforms of the scattering functions in the energy domain, $S(Q, t)=\mathcal{F}\left[I_{\text {expt }}(Q, \omega, T)\right] / \mathcal{F}\left[I_{\text {expt }}(Q, \omega, 10 \mathrm{~K})\right]$ [57], present the two well separated regions that were also previously observed in NS experiments on PC and PSF. As in those studies, the first steep decay at $t \leqslant 2$ ps in PHd6 would be ascribed to vibrational and "fast" processes. As the interpretation of these processes is beyond the scope of this work, we have characterized the decay at times shorter than

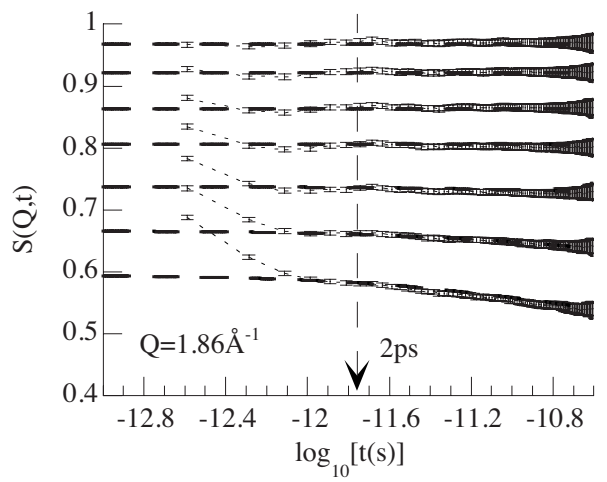

FIG. 4. Intermediate scattering functions $S(Q, t)$ of PHd6 in the microscopic time scale at $Q=1.86 \AA^{-1}$ and temperatures from 50 (top) to $350 \mathrm{~K}$ (bottom) every $50 \mathrm{~K}$. Dotted lines are guides for the eye between experimental points, which are represented by the corresponding error bars. Dashed lines are the intermediate scattering functions calculated with the $\pi$ flip plus short-time motion model. 

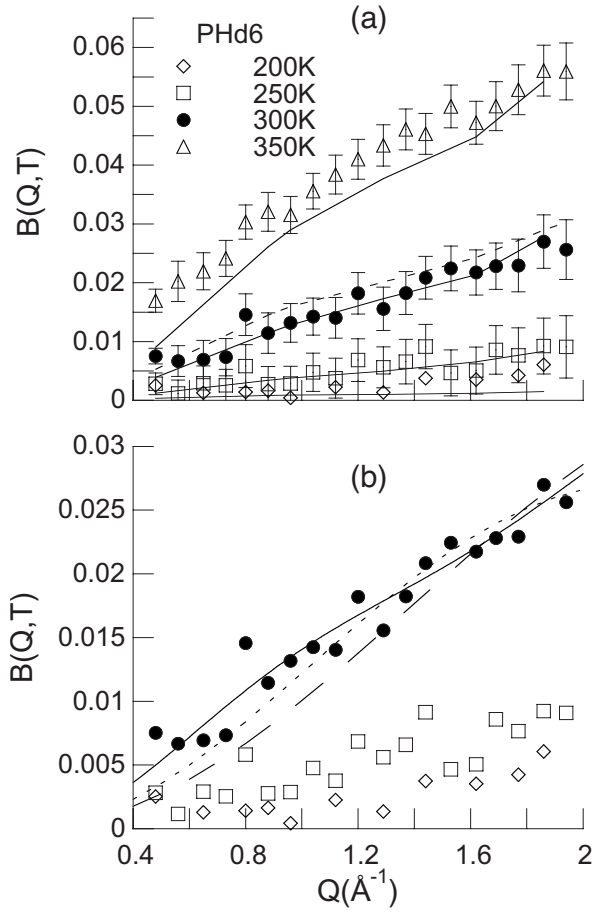

FIG. 5. (a) $B(Q, T)$ obtained from TOF experiments in the PHd6 sample as a function $Q$ and at different temperatures. Solid lines represent the predictions for the $\pi$ flip plus short-time motion model. The dashed line represents the prediction for the $\pi$ flip, plus short time, plus the slow $H_{c}$ motion model. (b) Details of the $300 \mathrm{~K}$ data. The dotted and dashed lines represent the $[1-A]$ function's $Q$ dependence for $d=2 \AA$ and $d=1 \AA$, respectively. The solid line represents the $\left(1-A_{\text {short }}\right)+0.08 A_{\text {short }}\left(1-A_{\text {flip }}\right)$ function $Q$ dependence resulting from $\pi$ flip plus a short-time motion of small amplitude geometry when $\tau_{\text {short }} \ll \tau_{\text {flip }}$ (see Appendix B). These geometric functions have been scaled so that the high- $Q$ experimental data level is matched.

$\sim 2$ ps by an effective Debye-Waller factor $\left(D_{e f f}\right)$. For a first characterization of the second step at $t \gtrsim 2 \mathrm{ps,} \mathrm{we} \mathrm{have} \mathrm{cal-}$ culated the slope of the $S(Q, t) / D_{\text {eff }}$ function vs $\log _{10} \mathrm{t}$ at $t$ $\geq 2$ ps for different temperatures (i.e., essentially the slopes of the curves at $t>2$ ps in Fig. 4),

$$
\left|\frac{d S(Q, t)}{d \log _{10} t}\right| \frac{1}{D_{\text {eff }}}=B(Q, T) .
$$

For a localized motion this quantity is nearly proportional to the $[1-A(Q)]$ function, being $A(Q)$ the elastic incoherent structure factor [24]. For jumps between two equivalent positions separated by a distance $d,[1-A(Q)]=1 / 2$ $-\sin (Q d) / 2 d$, so that the position of the first maximum locates at $Q d=3 \pi / 2$. The experimental $B(Q, T)$ values for different temperatures depicted in Fig. 5 show no maxima below $Q=2 \AA^{-1}$ indicating predominant small amplitude motion for aromatic and aliphatic hydrogens in this time scale. This question will be addressed later in Sec. IV.

\section{IN16 measurements: The mesoscopic time scale}

The spectra measured for PHd11 and PHd6 in the mesoscopic time scale by means of the BS IN16 instrument also

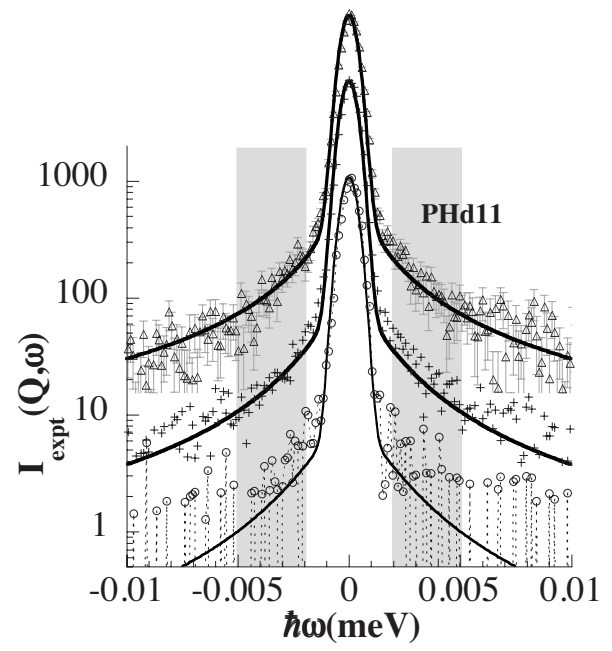

FIG. 6. PHd11 spectra in the mesoscopic time scale at $Q$ $=1.86 \AA^{-1}$ and $200 \mathrm{~K}$ (circles), $250 \mathrm{~K}$ (crosses), and $300 \mathrm{~K}$ (triangles). All the spectra are plotted in the same logarithmic scale, but have been shifted in the " $y$ " axis for clarity $(250 \mathrm{~K}, 0.8$ and $300 \mathrm{~K}, 1.3$ decades, respectively). The scattering of the data is indicative of the error. As an example error bars for $300 \mathrm{~K}$ have also been depicted. Solid lines represent the intensity calculated by the model function proposed. The dotted line in $200 \mathrm{~K}$ is a guide for the eye between experimental points evidencing the points below the axis. The shadowed area represents the quasielastic window considered in the definition of $a 2(Q, T)$ (see the text).

exhibit increasing quasielastic intensity as the temperature increases indicating the occurrence of some motion within this time scale (see Fig. 6). The increase of the quasielastic broadening with temperature can better be noticed looking at the integrated quasielastic intensity in a given energy window. The $Q$ dependence of the quasielastic intensity integrated between 2 and $5 \mu \mathrm{eV}$ (shadowed area in Fig. 6), $a 2(Q, T)$, normalized to the whole area of the spectra $a(Q, T)$ is depicted in Fig. 7 for PHd11 and PHd6. Double scale is such that quasielastic intensities normalized to $\frac{\sigma_{\text {inc }}}{\sigma_{\text {tot }}}$ are compared. As can be seen, the quasielastic scattering of PHd11 and PHd6 in the IN16 window are very similar. The quasielastic intensity (also normalized to $\frac{\sigma_{\text {inc }}}{\sigma_{\text {tot }}}$ ) for PSF at $300 \mathrm{~K}$ is also represented by crosses for comparison.

\section{PHENYLENE RING DYNAMICS IN PH}

Based on the qualitative analysis of the NS data in both microscopic and mesoscopic time scales and taking advantage of the experience acquired in the description of phenylene ring dynamics on PSF and PC together with previous literature results, we will in the following build up a model scattering function describing PH's neutron scattering. According to previous NS results the phenylene rings of both PSF and PC perform fast oscillation with such characteristic times that this motion is the main (but not the only) contribution to the TOF window signal at $t>2 \mathrm{ps}$, and also contributes to IN16 quasielastic intensity at low temperatures. Deuterium NMR measurements also indicate [7] that there exists a librational motion of phenylene rings in $\mathrm{PH}$ but, to 

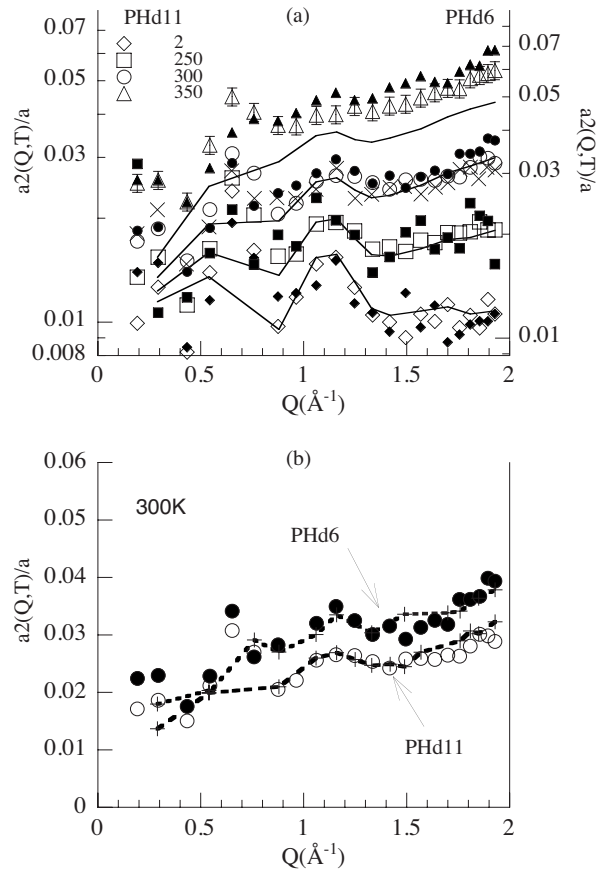

FIG. 7. (a) $Q$ dependence of $a 2(Q, t) / a$ in the IN16 window for PHd11 (left scale-empty symbols) and PHd6 (right scale-filled symbols), at several temperatures. The error is between 5 and $6 \%$, i.e., approximately the size of the symbols, as indicated for the highest temperature. Solid lines represent the predictions of the $\pi$ flip plus short-time motion model for PHd11. Crosses: PSF $a 2(Q, t) / a$ at $300 \mathrm{~K}$. (b) Same as in (a) for $300 \mathrm{~K}$. Plus signs linked by dashed lines represent the prediction for the $\pi$ flip, plus short time, plus slow $H_{c}$ motion model.

our knowledge, no quantitative characterization of the frequency of this motion has been performed. On the other hand, and regarding slower motions, $D$-NMR measurements [7] indicate that phenylene rings in $\mathrm{PH}$ perform $\pi$-flip motion with characteristic times, which are in very good agreement with those observed for the $\pi$-flip motion of phenylene rings in PSF and PC by NS (see below). In addition, and also according to D-NMR measurements, trans-gauche isomerization of methylene units occurs with mean characteristic time $(\tau=1 / 2 \pi f) 10^{-6} \mathrm{~s}$ at $300 \mathrm{~K}$.

As mentioned before, TOF measurements were performed in a PH sample with d6 deuteration, while IN16 measurements were conducted on both deuterations d6 and d11. Concerning the study of phenylene ring dynamics the PHd11 sample would be the most appropriate one. At the moment we have no data available in the microscopic time scale for PHd11, but taking advantage of the selective deuteration of PHd6 and PHd11 additional information can be obtained from the comparison of the corresponding scattered intensities. While the motion of all hydrogens except those in methyl groups is manifested in the quasielastic scattering of PHd6, the quasielastic signal in PHd11 is predominantly due to the motion of aromatic hydrogens $H_{a r}$. Comparing $a 2(Q, T) / a$ for the two samples (Fig. 7), it is concluded that hydrogen atoms in the aliphatic chain $H_{c}$ also move. The reason is the following. The magnitude of the $a 2(Q, T) / a$ function is approximately proportional to the moving hydro- gen's scattering cross section relative to the total scattering cross section $\frac{\sigma^{H}}{\sigma^{t o t}}$. If only $H_{a r}$ were mobile, $\frac{\sigma^{H}}{\sigma^{t o t}}$ would be around 0.49 and 0.68 for PHd6 and PHd11, respectively implying more quasielastic intensity for the PHd11 sample. This is not experimentally observed (note the double scale in Fig. 7). Regarding short-time motion of $H_{a r}$ and $H_{c}$, even if we have no PHd11 data in the TOF window to directly compare both samples, previous experience shows us that some information can be inferred from the low temperature $(200-300 \mathrm{~K})$ quasielastic intensity in the mesoscopic time scale $[13,14,17]$. At the lowest temperatures, the contributions of slower motions to the IN16 window are still small or even negligible, so that the quasielastic intensity measured is mainly produced by the short-time motion observed in the microscopic time scale. As a consequence, and following the same reasoning as before, both $H_{a r}$ and $H_{c}$ move in the microscopic time scale and contribute to the PHd6's TOF quasielastic signal at $t>2 \mathrm{ps}$. Moreover, at the lowest temperatures the IN16 quasielastic intensities of PHd6 and PHd11 normalized to $\frac{\sigma^{H}}{\sigma^{\text {tot }}}$ are very similar (see Fig. 7), indicating that the short-time motion of hydrogens in aromatic and aliphatic positions is similar (see Appendix A). As a result, in the following section we will assume the short-time motion of $H_{a r}$ and $H_{c}$ to be the same. We want to emphasize that, according to the low temperature BS data, $H_{c}$ must move, and its short-time motion cannot be considerably "slower" than that of $H_{a r}$, or PHd11 would have higher IN16 quasielastic intensity at low temperatures than PHd6.

Analyzing the experimental data, from Figs. 4-7 it is clear that in $\mathrm{PH}$ some motion (of one type or more) takes place at the time scales and temperatures indicated. The $Q$ dependence in Fig. 5 is similar to that found for PC and PSF in the same time scale and is evidence that the main contributing motion in the TOF window at $t>2 \mathrm{ps}$ is of small amplitude, as was the case for PSF and PC. On the other hand, the quasielastic intensity of PHd11 in the IN16 window at $300 \mathrm{~K}$ is very similar to that of PSF (see the crosses in Fig. 7 ). We note that the results obtained above from the qualitative analysis of PHd6 and PHd11 neutron scattering are compatible with the information in the literature. In principle, the short-time motion of $H_{a r}$ and $H_{c}$ observed in the TOF window would be consistent with the oscillation motion of phenylene rings in Refs. [13,14,17] and with the fast librations of methylene groups in Ref. [7]. On the other hand, the slower (high temperature) contributions of $H_{a r}$ and $H_{c}$ to the quasielastic intensity in IN16 could be due to phenylene ring $\pi$ flips and trans-gauche isomerization, respectively.

\section{Quantitative description of the data}

The experimental scattered intensities of $\mathrm{PH}$, both in the microscopic and mesoscopic time scales, have been described by means of a model scattering function, which considers short-time and slow motions for its hydrogens. In the construction of the model, motions are considered statistically independent and to have distributed characteristic times, which originate from the packing heterogeneity produced by the amorphous character of polymers. Accordingly, 


$$
S_{H}^{i n c}(Q, t)=\sum_{k} p_{k} S_{\text {slow }}^{k}(Q, t) S_{\text {fast }}^{k}(Q, t)
$$

where

$$
\sum_{k} p_{k}=1
$$

and

$$
S_{\alpha}^{k}(Q, t)=A_{\alpha}+\left(1-A_{\alpha}\right) e^{-t / \tau_{\alpha}},
$$

with $\alpha=$ slow or fast. The weight $p_{k}$ would be determined by the distribution of certain undefined structural parameter $\xi$ driving $H$ dynamics. It is also to be expected that both the characteristic time $\left(\tau_{\alpha}\right)$ and the activation energy $\left(E_{a}^{\alpha}\right)$ depend on such structural parameter $\xi$, and therefore, $p_{k}$ can also be formally parametrized in terms of a distribution of $\tau_{\alpha}$ or $E_{a}^{\alpha}$ (see Refs. $[13,14]$ for further details),

$$
p_{k}=\int_{\delta \xi} g(\xi) d \xi=\int_{\delta \tau_{\alpha}} f\left(\tau_{\alpha}\right) d \tau_{\alpha}=\int_{\delta E_{a}^{\alpha}} f\left(E_{a}^{\alpha}\right) d E_{a}^{\alpha} .
$$

Regarding the $T$ dependence of the characteristic times, according to what is established for sub- $T_{g}$ processes an Arrhenius type $T$ dependence has been assumed,

$$
\tau_{\alpha}=\tau_{\infty} e^{E_{a}^{\alpha} / K_{B} T}
$$

with a preexponential factor $\tau_{\infty}=10^{-13} \mathrm{~s}$. Finally, although the incoherent scattering of $H$ dominates the measured scattering cross section, all the coherent scattering and the incoherent scattering of atoms other than $H$ have also been taken into account in the model function by adding the contribution of these signals as elastic terms in a first approximation,

$$
\begin{aligned}
I^{\text {model }}(Q, t)= & D_{e f f}\left[\left(\frac{d \sigma}{d \Omega}\right)_{c o h}+\sigma_{i n c}^{\text {other }}+\sigma_{i n c}^{H_{a r}} S_{H_{a r}}^{i n c}(Q, t)\right. \\
& \left.+\sigma_{i n c}^{H_{c}} S_{H_{c}}^{i n c}(Q, t)\right],
\end{aligned}
$$

where $S_{H_{a r}}^{i n c}(Q, t)$ and $S_{H_{c}}^{i n c}(Q, t)$ are defined as in Eq. (2), and $\left(\frac{d \sigma}{d \Omega}\right)_{c o h}$ is taken from D7 measurements results. In order to compare the model and experimental functions, $I^{\text {model }}(Q, t)$ has been normalized to its static (low $T$ ) value, whereas in the energy domain $I^{\text {model }}(Q, \omega)$ [the energy counterpart of Eq. (7)] has been convoluted with the experimental resolution.

In line with the previous PSF and PC studies, first we checked that also in $\mathrm{PH}$ the short-time motion dominating the TOF signal at $t>2$ ps notably contributes to the quasielastic intensity in the IN16 window, but cannot by itself give account for the whole IN16 quasielastic intensity (especially at high temperatures). This result indicates the presence of an additional slower motion for $H$. When a single motion model is used to fit the data in the TOF window at $t>2 \mathrm{ps}$, the amplitude of the motion $(\sim 2 \AA)$ and the mean activation energy giving a satisfactory description are comparatively higher than those reported in the literature for phenylene ring fast oscillations in PSF and PC. The consideration of amplitudes similar to those in the literature for this short-time motion results in deficient intensities (slopes) at intermediate $Q$ values (see the $[1-A]$ function for $d=1 \AA$ in
Fig. 5). The lack of TOF intensity in such a $Q$ range when using short-time motion amplitudes similar to those in the literature, suggests that the additional motion could be $\pi$ flips of phenylene rings. The reason is that due to the maximum of the $[1-A]$ function for $\pi$ flips at $Q \sim 1 \AA^{-1}$, they are a good candidate to, together with fast motions of small amplitude, reproduce the experimentally observed $Q$ dependence in the microscopic time scale. The approximated $Q$ dependence (see Appendix B and Ref. [13]) for such a case is depicted in Fig. 5 with a solid line. The fact that this was also the situation when we tried to fit the PSF data in terms of only one motion, and that the IN16 quasielastic signal for PHd11 and PSF at $300 \mathrm{~K}$ are almost indistinguishable (see the crosses in Fig. 7) support the presence of $\pi$ flips also for $\mathrm{PH}$ phenylene rings.

Based on these results and according to the literature we assumed the extra slow motion to be $\pi$ flips of phenylene rings. Thus, on a second approach two different motions with distinct mean characteristic times were considered: (i) fast small amplitude motions of phenylene rings and aliphatic hydrogens, and (ii) $\pi$ flips of phenylene rings. In principle, by means of Eq. (7) and taking into account that the contribution of the $\pi$-flip motion to the TOF window at high $Q$ s is small and the contribution of short-time motion to the IN16 window at intermediate $Q$ s is minimized [13], it would be possible to determine the dynamic parameters for the shorttime and $\pi$-flip motions for $\mathrm{PH}$, as was previously done for PSF. However, in practice, the determination of $\pi$-flip activation energy distribution from PHd11 IN16 data is to some extent uncertain, because the low $T_{g}$ of the polymer together with the slow character of $\pi$ flips reduce the number of measured temperatures where the $\pi$-flip motion contribution starts to be noticeable to a single one, i.e., $300 \mathrm{~K}$. As a consequence, the fitting procedure in order to analyze the data in terms of two motions was similar to that explained in Ref. [13], but fixing the parameters for the $\pi$-flip motion to values close to those in the literature. In any case, even if the fitting of the PHd11 data per se cannot give an accurate activation energy for $\pi$-flip motion, not all the conceivable activation energy distributions produce a satisfactory description of the experimental data, and therefore, the condition of consistence between the calculated and measured scattering will be an additional test and support for the validity of the fixed parameters.

Figures 4-7 show the predictions of the short-time plus $\pi$-flip motion model for PHd11 BS and PHd6 TOF data. As can be seen, the calculated magnitudes satisfactorily describe the experimental features observed. At the highest temperature measured, calorimetric data indicate that glass transition has already begun. This, together with the lack of a proper model for $H_{c}$ slow motion (see below), is the reason for the slightly lower calculated intensities at $350 \mathrm{~K}$. The mean value and width (HWHM) of the distribution of activation energies for the short-time motion providing the mentioned good description of the experimental data are $\left\langle E_{a}^{o s c}\right\rangle$ $=0.21 \mathrm{eV}$ and $\mathrm{HWHM}_{\text {osc }}=0.081 \mathrm{eV}$, respectively. The mean amplitude of the short-time motion is found to increase with temperature. Parametrized in terms of oscillations around the equilibrium position, the mean oscillation angle $\langle\theta\rangle$ was $6^{\circ}$, $12^{\circ}$, and $20^{\circ}$ at 200,250 , and $300 \mathrm{~K}$, respectively. 
TABLE I. Mean activation energies and widths (HWHM) of the distributions obtained by NS for oscillation, $\pi$ flip, and extra motion (only PC) in PH, PSF, and PC. The jump distance for phenylene ring flips is imposed by the geometry of the molecule and is $d=4.3 \AA$. $\left({ }^{*}\right.$ ) stands for phenylene ring oscillations mean jump distance $\langle d\rangle=4.3 \sin (\langle\theta\rangle / 2)$ being $\langle\theta\rangle$ the mean amplitude of the oscillation. Estimated error for HWHM and $d$ are $\pm 0.005 \mathrm{eV}$ and $\pm 0.1 \AA$, respectively.

\begin{tabular}{lcccccccc}
\hline \hline & $\begin{array}{c}\left\langle E_{a}\right\rangle_{\text {osc }} \\
(\mathrm{eV})\end{array}$ & $\begin{array}{c}\mathrm{HWHM}_{\text {osc }} \\
(\mathrm{eV})\end{array}$ & $\begin{array}{c}\left\langle E_{a}\right\rangle_{\text {flip }} \\
(\mathrm{eV})\end{array}$ & $\begin{array}{c}\mathrm{HWHM}_{\text {flip }} \\
(\mathrm{eV})\end{array}$ & $\begin{array}{c}\left\langle E_{a}\right\rangle_{\text {rot }} \\
(\mathrm{eV})\end{array}$ & $\begin{array}{c}\mathrm{HWHM}_{\text {rot }} \\
(\mathrm{eV})\end{array}$ & $\begin{array}{c}d_{\text {short }}^{*}(300 \mathrm{~K}) \\
(\AA)\end{array}$ & $\begin{array}{c}d_{\text {short }}^{*}(250 \mathrm{~K}) \\
(\AA)\end{array}$ \\
\hline PH & $0.21 \pm 0.01$ & 0.081 & $0.41 \pm 0.02$ & 0.013 & & & 0.7 & 0.4 \\
PSF & $0.20 \pm 0.01$ & 0.086 & $0.43 \pm 0.02$ & 0.012 & & & 0.8 & 0.70 \\
PSF & $0.21 \pm 0.01$ & 0.086 & $0.41 \pm 0.02$ & 0.013 & $0.32 \pm 0.01$ & 0.09 & 0.8 & 0.70 \\
\hline \hline
\end{tabular}

When the short-time plus $\pi$-flip motion model is used to predict the quasielastic signal of PHd6 in the IN16 window the calculated intensities lie slightly below the experimentally observed values (especially at high temperatures). This suggests the presence of an additional slow motion for $H_{c}$, which would be in agreement with the trans-gauche isomerization of methylene units proposed by Jones et al. [7]. As was the case for $\pi$-flip motion, the determination of the activation energy distribution and geometry for this $H_{c}$ slow motion is not feasible from our NS data. The lack of this information prevented us from calculating the whole PHd6 quasielastic signal in the IN16 window. We may ask what would be the influence of considering this $H_{c}$ slow motion on the results obtained for the other motions. The low relative scattering weight of the single $H_{c}$ in PHd11, and the small contribution to the TOF window of the slow motion of $H_{c}$, indicate that the results obtained from PHd6 TOF and PHd11 IN16 data by the $\pi$-flip plus short-time motion model should be quite good. Nevertheless, in order to evaluate this influence we have modeled the $H_{c}$ slow motion with more or less realistic parameters such that the calculated quasielastic intensities for PHd6 in the IN16 window are well compatible with the experimental ones shown in Fig. 7. Figures 5 and 7 show the results of considering now this $H_{c}$ slow motion in the description of PHd6 TOF and PHd11 IN16 data. As can be seen, the influence is small and below the error in both cases. Either the increment of the mean oscillation angle $\langle\theta\rangle$ of short-time motion in $1^{\circ}$ or the consideration of the $H_{c}$ slow motion, have approximately the same influence on the calculated $B(Q, T)$ intensity at $300 \mathrm{~K}$. Therefore, to take into account the $H_{c}$ slow motion would modify the results for the mean oscillation angle $\langle\theta\rangle$ of short-time motion at $T$ $\geq 300 \mathrm{~K}$ just by $1^{\circ}$.

\section{DISCUSSION}

One of the reasons to perform a detailed study of the molecular motions of phenylene rings was to shed new light on the presumed relation between their dynamics and the $\gamma$ relaxation, and to try to answer the question about the molecular origin of the $\gamma$ relaxation in this type of polymer. Our approach is to (i) characterize phenylene ring dynamics by NS in PSF, PH, and PC looking for differences and similarities, and (ii) try to relate the molecular motions identified by NS with the secondary relaxations observed by spectroscopic techniques published throughout the literature for these three systems. These two steps will be given in the next sections. As we will see, there exists a good correlation between the $\gamma$ relaxation (both mechanical and dielectric) of PH, PSF, and PC and the motion of their constituent molecular groups.

\section{A. Motions identified for phenylene rings}

In this section, a summary of phenylene ring dynamics in PH, PSF, and PC will be presented with emphasis on the differences and similarities found between the sub- $T_{g}$ dynamics of the three polymers.

\section{Oscillations}

In the three polymers considered a relatively fast motion of small amplitude and broadly distributed characteristic times has been identified. This motion is mainly responsible for the observed second decay of the $S(Q, t)$ functions in the microscopic range. Though it is mainly centered in the microscopic time scale at $T \sim 300 \mathrm{~K}$, its contribution to the mesoscopic scale at low temperatures has also been proved to be important. The mean activation energies obtained for this motion, characterized as oscillations of phenylene rings $\left\langle E_{a}^{o s c}\right\rangle$, were essentially the same for the three polymers, $0.20 \pm 0.01 \mathrm{eV}$ for PSF and PC, and $0.21 \pm 0.01 \mathrm{eV}$ for PH (see Table I). The obtained values are within the range $0.16-0.23 \mathrm{eV}$ estimated by deuterium NMR in PC [25] and also agree well with the activation energy obtained by NS for phenylene ring oscillations in polyethersulfone [17]. As already mentioned in our previous works [13-15], $\left\langle E_{a}^{o s c}\right\rangle$ would not represent a real potential barrier over which a phenylene ring jumps, but a measure of the rapid fluctuations of the local rotational phenylene ring potential. These fluctuations would arise as a consequence of small amplitude motions provoked by bond motions, thermal fluctuations, and small atomic rearrangements (accommodations) in response to larger molecular motions. Thereby, the distribution of activation energies or equivalently, the distribution of characteristic times for oscillations would reflect the characteristic times of all the fluctuations perturbing the rotational potential of phenylene rings. We note that the distribution of activation energies for methyl group rotation in these systems covers a notable portion of the distribution of $E_{a}^{o s c}$ [26]. This would be indicative of a significant contribution of methyl group motion to the fluctuations of the local rotational potential in the particular case of phenylene rings within 

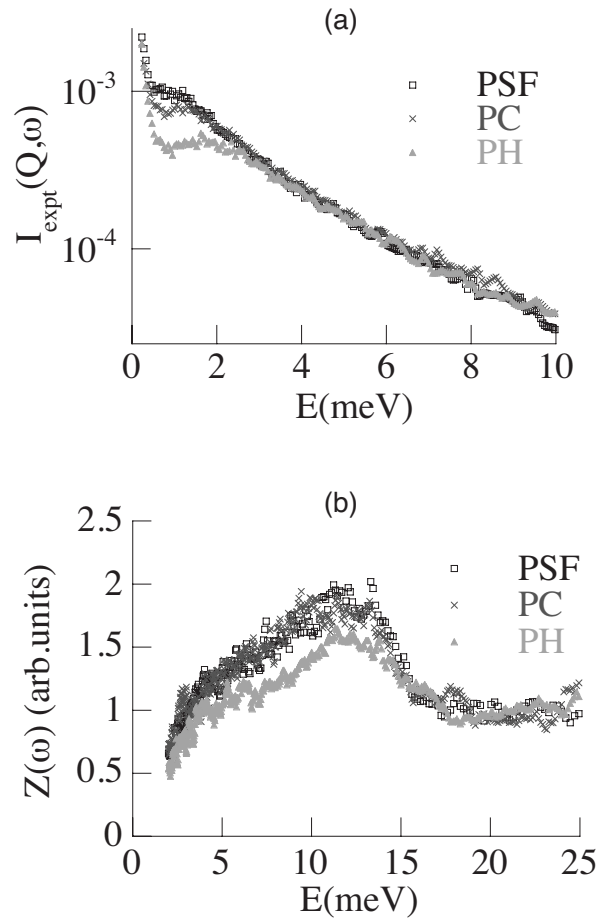

FIG. 8. (a) Experimental spectra normalized to the maxima of the elastic intensity in the TOF window for PSF, PC, and PH, at $50 \mathrm{~K}, Q \sim 1 \AA^{-1}$, and at logarithmic scale. (b) $H$-weighted density of states normalized to the high energy value, by TOSCA $[26,34]$.

BPA units, in agreement with previous suggestions in the literature [27,28].

Though the value of $\left\langle E_{a}^{o s c}\right\rangle$, or mean characteristic times, obtained from the analysis are similar for the three polymers, differences have been found in the amplitude of these oscillations around the equilibrium position, i.e., on $\langle\theta\rangle$. The $\langle\theta\rangle$ values in PC and PSF are very similar, whereas in $\mathrm{PH}$ they are slightly smaller at low temperatures: $\langle\theta\rangle$ is equal to $6^{\circ}$, $12^{\circ}$, and $20^{\circ}$ at 200,250 , and $300 \mathrm{~K}$ in $\mathrm{PH}$, in contrast to $18^{\circ}, 19^{\circ}$, and $21^{\circ}$ in PSF or PC. The smaller amplitude of oscillations in $\mathrm{PH}$ would indicate more restricted mobility for this polymer in comparison with the two others. A better packing of the local structure in $\mathrm{PH}$ due to the higher flexibility of the linking group between BPA units, the aliphatic chain, could be the origin of this reduced amplitude.

It is interesting to note that the mean square displacement, associated to the amplitude of the fast motions in the microscopic time scale (see the inset of Fig. 3) also exhibits marked differences between PH on the one hand, and PC and PSF on the other [29], showing smaller amplitude in PH. In addition, we observe a higher characteristic frequency for the boson peak in $\mathrm{PH}$ as compared with those observed for PC and PSF, and a lower density of states in the low frequency range for $\mathrm{PH}$ than for PSF or PC (see Fig. 8). These results would also support more restricted mobility and better packing of the structure in $\mathrm{PH}$ in light of recent researches [30-33]. Frick et al. showed that an increase of pressure (and presumably of packing) in glass-forming systems produces a shift of the boson peak to higher frequencies and a subsequent decrease of the low frequency modes in the density of states, together with a decrease of the mean square displace- ment [30-32]. Soles et al. on the other hand, found an inverse correlation between the size of the free volume measured by means of positron annihilation lifetime spectroscopy, and the boson peak, so that the frequency of the peak increases as the free volume decreases [33]. At the sight of these results, the effect of the aliphatic chain as a linking group between BPA units resembles the plastization and antiplastization of polymers by the addition of low molecular compounds or blending. On the one hand, the higher flexibility of the aliphatic chain in $\mathrm{PH}$ makes its $T_{g}$ considerably lower than those of PC or PSF, in some sort of "plastization" effect. On the other hand however, the mobility below $T_{g}$ is restricted resulting in an "antiplastization" of the polymer.

\section{2. $\pi$ flips}

The $\pi$-flip motion, observed in the three polymers investigated, would be quite similar in all cases: a motion mainly detected in the mesoscopic window at high $T$ with characteristic times distributed over several orders of magnitude. The mean activation energies for $\pi$-flip motion $\left\langle E_{a}^{f l i p}\right\rangle$ were $0.43 \pm 0.01 \mathrm{eV}$ for PSF, and $0.41 \pm 0.01 \mathrm{eV}$ for PC and PH. These values are in good agreement with those obtained for $\pi$-flip motion by NMR $[3,4,7,8]$. Phenylene rings in PC and $\mathrm{PH}$ are equivalent (they are within the same molecular group, the BPA moiety), whereas PSF contains two types of rings (within the BPA group and within the sulphone group), which were assumed to be equivalent in the analysis of the NS data. The fact that the activation energies obtained for all the polymers are essentially the same supports the results in Ref. [5], where evidence of indistinguishable motion for all phenylene rings in PSF by NMR was given. In any case, little differences could be attributed to different intramolecular and intermolecular environments for the rings. It is known that PR- $X$-PR- $(\mathrm{PR}=$ phenylene ring) type structures involve relatively high intramolecular coupling between the motion of the rings surrounding $X$, providing $X$ is simple enough [35-42]. A higher concentration of phenylene rings along the chain in PSF could be the reason for the slightly higher energy observed, via the mentioned higher intramolecular correlation. This idea is additionally supported by the likewise slightly higher $\left\langle E_{a}^{f l i p}\right\rangle=0.46 \mathrm{eV}$ found in polyethersulfone, a polymer that consists of the repetition of diphenylsulfone and diphenylether groups [17].

\section{Additional motion in PC}

Our NS investigations on PC showed that phenylene rings in the PC exhibit, in addition to $\pi$ flips and oscillations, extra quasielastic intensity at $T>300 \mathrm{~K}$ associated to a motion of amplitude around $3 \AA$ (see Fig. 9 and Refs. [14,16]). Likewise, extra motion of the rms amplitude of $\pm 35^{\circ}$ at $380 \mathrm{~K}$ has also been reported from NMR studies [3]. We interpreted this additional motion as rotations of about $90^{\circ}$ likely linked to the motion of the carbonate group $[14,16,18,38,43]$. The mean activation energy for this extra motion lays between the values for oscillations and $\pi$ flips, and is equal to $0.32 \pm 0.01 \mathrm{eV}$. This value would be in agreement with the simulations of Hutnik et al. [37] and Chen et al. [44], which 


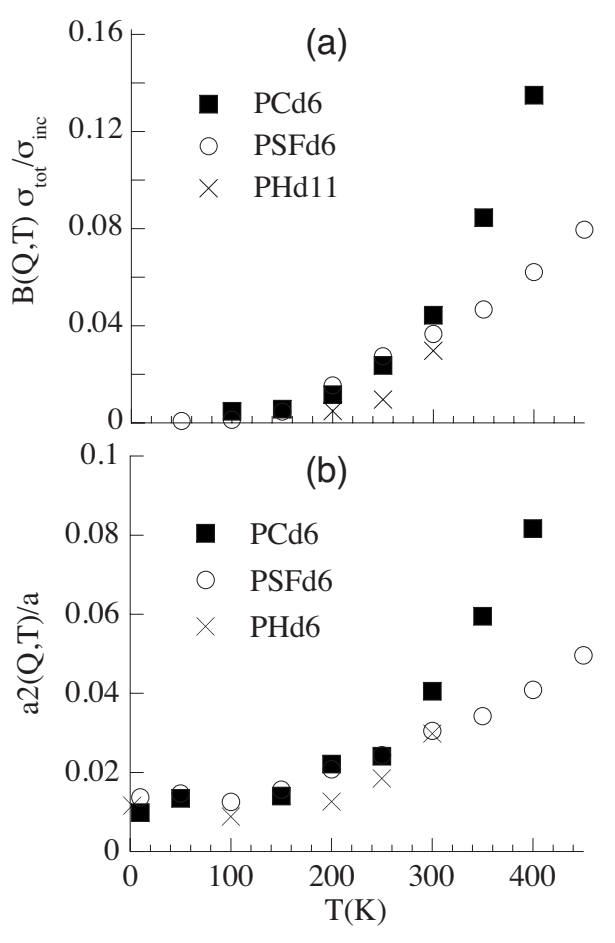

FIG. 9. a) $B(Q, T) \frac{\sigma_{\text {tot }}}{\sigma_{\text {inc }}}$ for PH, PSF, and PC at $Q=1.77 \AA^{-1}$ in the microscopic time scale (TOF). (b) $a 2(Q, T) / a$ for PH, PSF, and PC at $Q=1.89 \AA^{-1}$ in the mesoscopic time scale (IN16).

predict slightly more frequent rotations for the carbonate group (likely linked to the extra motion of phenylene rings in PC) than for phenylene $\pi$ flips. The higher mobility of phenylene rings in $\mathrm{PC}$ in comparison to rings in $\mathrm{PSF}$ or $\mathrm{PH}$ constitutes a good example of the possible effect of a specific linking group between BPA units on ring dynamics.

\section{B. Relation between ring dynamics and $\gamma$ relaxation}

The comparison of the results for phenylene ring dynamics by NS and $\gamma$ relaxation in PC were already published in previous communications $[14,16,18,45]$. In those works we showed how the characteristic times for phenylene ring $\pi$ flips and for the extra motion correlate with the times for the two components observed in PC's dielectric relaxation, which addresses the carbonate group motions. In addition, the significant difference observed between the position of the maxima of the $\gamma$ relaxation seen by dielectric and mechanical spectroscopy could be easily explained arguing that, among the two processes composing the relaxation, DS is more sensitive to the "fast" component (related to the extra motion times), while the motions responsible for the "slow" component (related to the ring $\pi$-flip times) would be more coupled to a mechanical field. Consistently, the characteristic times for the maxima of the mechanical $\gamma$ relaxation of PC correspond to the mean characteristic times for $\pi$ flips, whereas the characteristic times for the maxima of the dielectric $\gamma$ relaxation relate to those for the extra motion of PC rings. Additional work on the dielectric $\gamma$ relaxation of PC shows that as a result of cold drawing the fast component of the $\gamma$ relaxation related to the extra motion is more mark-

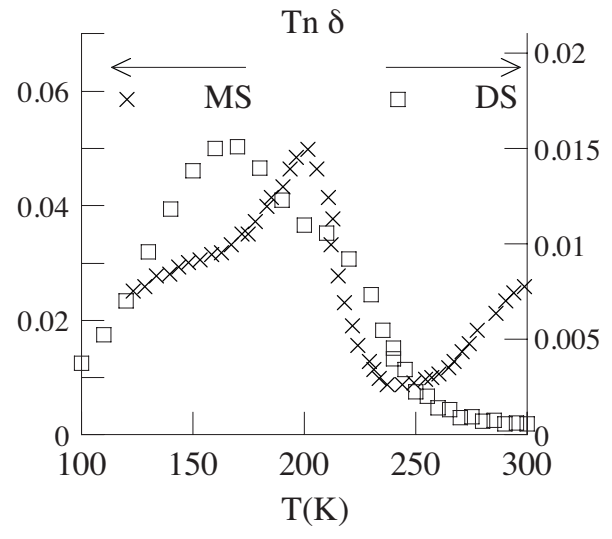

FIG. 10. Mechanical (crosses from Ref. [47]) and dielectric (squares) loss tangent for $\mathrm{PH}$ at $1 \mathrm{~Hz}$.

edly reduced than that reflecting the $\pi$-flip motion [18], which allowed one to unambiguously separate the different components of the relaxation. This effect has been tested and supported by NS measurements, which studied the effect of cold drawing on the motion of phenylene rings in BPA-PC [46].

Regarding the $\gamma$ relaxation in $\mathrm{PH}$, as in $\mathrm{PC}$, it would also consist of more than one molecular process. On the one hand, the positions of the maxima of the $\gamma$ relaxation observed in PH by dielectric and mechanical spectroscopy also differ from each other (see Fig. 10). Moreover, the mechanical relaxation quite clearly exhibits a "shoulder" at the position of the dielectric maximum and at the same time, the dielectric relaxation presents another "shoulder" around the position of the mechanical relaxation maximum (see Fig. 10). Takahama et al. in Ref. [48] proposed that the main peak in the mechanical relaxation of $\mathrm{PH}$ is due to aliphatic chain motions, and that the shoulder is due to motions of the diphenylpropane group. This is deduced from the observation that the intensity of the maximum decreases while that of the shoulder remains almost the same as a result of diminishing the concentration of hydroxiether groups (through acetylation). The idea of Takahama et al. is consistent with the NMR work of Jones et al. about the motion of the aliphatic chain in PH [7]. Figure 11 shows the characteristic times obtained by Jones et al. for the motion of the aliphatic chain (diamonds [7]), together with the times for the position of the maxima in the dielectric and mechanical relaxation, and the times for $\pi$-flip motion found by NS [58]. The extrapolation of the NMR times for the motion of the aliphatic chain (continuous line) coincides with the position of the maximum of the mechanical relaxation (full square [47]). On the other hand, the characteristic times of the maxima of the dielectric relaxation (empty squares), which would also represent the "shoulder" of the mechanical relaxation, are very close to those for ring $\pi$ flips (broken line), at least at low temperatures. In summary, the results are consistent with the idea that the $\gamma$ relaxation in $\mathrm{PH}$ consists of at least two processes, among which the slower one would be associated to the motion of the aliphatic chain, and the faster one to phenylene ring $\pi$ flips. The mechanical relaxation would be more sensitive to the motion of the aliphatic chain, whereas the dielectric relaxation would couple more efficiently to the motion of the rings. 


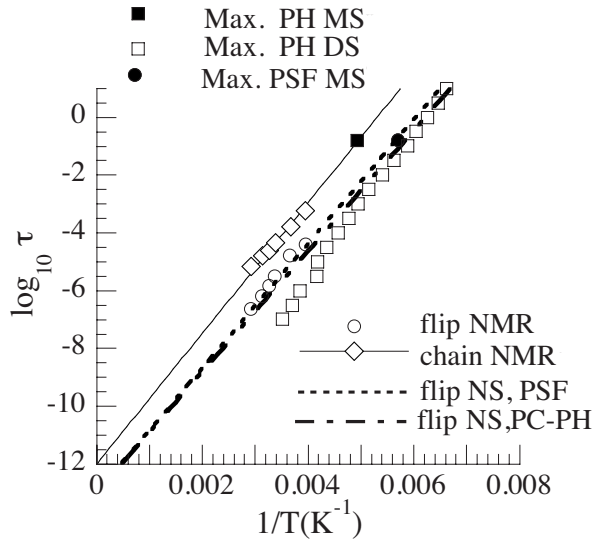

FIG. 11. Arrhenius plot for several characteristic times in PH and PSF. Broken line: times for $\pi$ flips in PH and PC obtained by NS. Dotted line: times for $\pi$ flips in PSF obtained by NS. Filled square: maxima of the mechanical relaxation in PH from Ref. [47]. Diamonds and empty circles: times for the motion of the aliphatic chain and phenylene ring $\pi$ flips by means of NMR from Ref. [7]. Continuous line: extrapolation of the times for aliphatic chain motion in PH. Empty squares: maxima of the dielectric relaxation in PH. Filled circle: maxima of the mechanical relaxation in PSF from Ref. [49].

Finally, the $\gamma$ relaxation in PSF presents some peculiarity, as the intensity of the relaxation (both mechanical and dielectric) notably depends on the humidity degree of the sample, and can even disappear (in the case of dielectric relaxation) when samples are subjected to severe drying treatment (see Fig. 12) [50]. In PSF the maxima of the mechanical and dielectric (in the presence of humidity) loss tangent do not differ from each other and coincide with (i) the position of the maxima of PC's mechanical loss, (ii) the position of the shoulder of PH's mechanical loss, and (iii) the position of the maxima of PH's dielectric loss. As a consequence, and as it was expected, the position of the maxima for the $\gamma$ relaxation in PSF is consistent with the mean characteristic times for $\pi$ flips (see the filled circle and dotted line in Fig. 11).

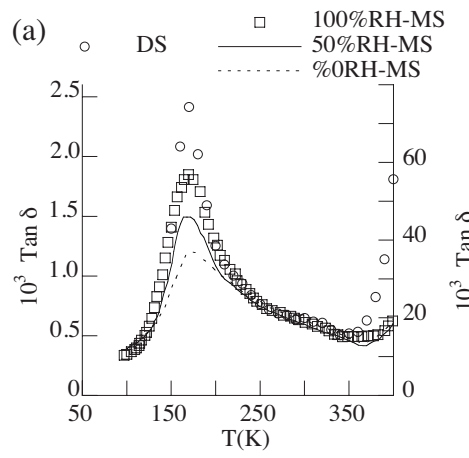

(b)

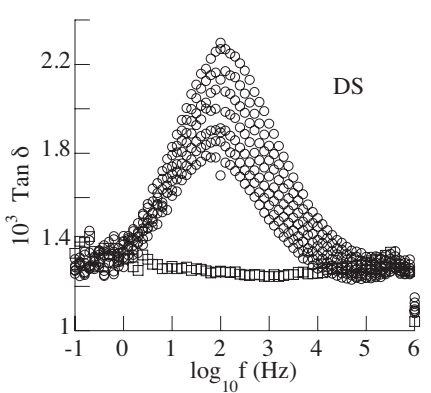

FIG. 12. (a) Dielectric (circles) and mechanical (squares and lines from Ref. [49], $\mathrm{RH}=$ relative humidity) loss tangent of PSF as a function of temperature at $1 \mathrm{~Hz}$. (b) Dielectric loss tangent of PSF as a function of frequency at $200 \mathrm{~K}$ for several humidity degrees (circles) and after desiccation (squares).

\section{Molecular motions in the secondary relaxation}

As we have seen, the various features of the $\gamma$ relaxation of $\mathrm{PC}, \mathrm{PH}$, and PSF can be well rationalized in terms of the addition of different contributions that are closely related to the motion of their constituent molecular groups. We want to emphasize the consistency of this analysis, which involves the results from various different techniques: NS, NMR, DS, and MS. Similarities in the phenylene ring dynamics of these systems are accompanied by similarities in their $\gamma$ relaxation. From a molecular point of view the three polymers exhibit nearly equal ring $\pi$ flips, while from a spectroscopic point of view they all have a common relaxation component at $170 \mathrm{~K}$ at $1 \mathrm{~Hz}$. Besides, the comparison of the experimental data from the two points of view leads to the result that both phenylene $\pi$ flips and the $170 \mathrm{~K}$ at $1 \mathrm{~Hz}$ component of the relaxation have the same mean characteristic times. Although phenylene ring $\pi$ flip itself cannot be the primary source of the relaxation, this is a clear indication (recurrent through literature) of the close relation existing between the two processes. The results reported here constitute an independent experimental evidence of the coincidence between $\gamma$ relaxation and phenylene ring motion times, which up to now was only revealed by NMR measurements. However, $\pi$ flips are not the only molecular motion reflected in the relaxation, and differences on the molecular motions in these three polymers also have their counterpart in the $\gamma$ relaxation. Thus, for each polymer the relaxation process also reflects the nature of the linking group between BPA units. A second relaxation component matches the characteristic time of (i) the aliphatic chain motion of $\mathrm{PH}$, and (ii) that of phenylene ring extra motion coupled to the motion of the carbonate group in PC. Consistently, the $\gamma$ relaxation in PSF, which has a PR- $X$-PR structure as a linking group between BPA units, does not seem to be composed of more than one process. In other words, the contribution of the linking group in PSF to the $\gamma$ relaxation would be indistinguishable from that of the BPA unit itself within the experimental accuracy of the technique. This is quite reasonable bearing in mind that we do not expect the behavior of the two PR- $X$-PR structures to differ considerably. Summarizing, we have seen that the nature of the linking group between BPA units influences the $\gamma$ relaxation so that new relaxation components arise that can be related to the motion of the linking group. Nevertheless, detailed information about the possible different in-chain cooperativity in these systems has not been obtained.

Yaris et al. [51,52] explained the relation between secondary relaxation and phenylene ring $\pi$-flip times by interchain cooperative effects. The argument is that a $\pi$ flip occurs when there is an increase in the separation distance between the ring and its nearest neighbor ring on another chain, allowing the ring to rotate. The simulations performed by Chen et al. show that a ring repels the neighboring rings to generate free volume for rotation and thereafter rings move closer to take the vacancy produced by the rotation $[38,44]$. This idea is well supported also by the experimental NMR work of Schaefer et al. [53-55] and by the interpretation of DS results of stretched PC recently reported by Mitxelena et al. [18]. Based on Yaris et al.'s idea, Horii et al. proposed an "open gate model" in which phenylene ring motions activate 
the main-chain motions, and the main-chain motions in turn make the rings $\pi$ flip [56]. According to them, the characteristic frequencies of main-chain fluctuations and phenylene ring motion coincide explaining the correlation between mechanic $\gamma$ relaxation and $\pi$-flip times. However, we have seen that secondary relaxation reflects the characteristic times of different molecular motions rather than only a single characteristic time for main-chain fluctuation. From our point of view, both main-chain fluctuations and the rest of the molecular motions identified (whose characteristic times relate to the $\gamma$ relaxation) are part of a complex and indivisible concerted mechanism leading to the observed $\gamma$ relaxation. The fact that some of the molecular motions identified, which are dielectrically or mechanically inactive (like symmetric $\pi$ flips) can leave their fingerprint on the secondary relaxation supports the above mentioned interpretation.

Regarding the amplitude of main-chain fluctuations in the systems studied, some conclusions can be drawn from the NS data. For a given characteristic time NS gives an upper bound to the length scale of main-chain fluctuations as they cannot be larger than the largest motion detected. The observed amplitude of ring oscillations was between 0.7 and $0.8 \AA$ at $300 \mathrm{~K}$, so main-chain fluctuations with times similar to phenylene ring oscillations should be smaller than or equal to those values. On the other hand, according to our NS results main-chain fluctuations with characteristic times similar to $\pi$ flips should be equal to or lower than $\sim 0.8 \AA$. The reason is that motions with such a slow characteristic time should manifest in the high temperature $Q$ dependence of the quasielastic intensity in backscattering experiments, where fast oscillations no longer significantly contribute to the measured quasielastic scattering [13]. However, the $Q$ dependence of the high temperature PSF data in the IN16 window is in very good agreement with a $\pi$-flip geometry, i.e., with the $[1-A]$ function for $\pi$ flips, so that the maximum amplitude for the main-chain motion compatible with the observed experimental $Q$ dependence is around $0.8 \AA$. This value is compatible with the $0.5 \AA$ increase in the interring distance observed by Yaris et al. [52] and with the $\sim 0.7 \AA$ displacement predicted by Chen et al. during a ring $\pi$ flip [38]. Both results were obtained by simulation techniques.

\section{CONCLUSIONS}

The NS of PHd6 and PHd11 in the microscopic and mesoscopic time scales, respectively, can be well described by a model that considers $\pi$ flips and oscillation motions for phenylene rings. The values of the mean activation energies for $\pi$-flip and oscillation motion, which provide the mentioned good description, are in good agreement with previous values obtained by NS for PSF and PC, and with those obtained by NMR for PH and PC. The smaller amplitude of the oscillation motion for phenylene rings in $\mathrm{PH}$ at low temperatures, the lower mean square displacement associated to the fast dynamics, and the higher energy of the boson peak, suggest more restricted mobility for this polymer in comparison with PSF and PC. A better packing of the local structure in PH due to the higher flexibility of the linking group between
BPA units (the aliphatic chain) could be the origin of this reduced mobility at lower temperatures. On the other hand, the hydrogens in the aliphatic chain also perform fast motions of small amplitude similar to those observed for the oscillations of phenylene rings. Moreover, experimental data of PHd6 support the presence of additional slow motion for these hydrogens.

As in the case of PC, the secondary relaxation in $\mathrm{PH}$ does not correspond to a single process but compresses different contributions that couple to dielectric or mechanical fields with different strengths. The characteristic times of the motions of different molecular groups within PH (phenylene ring and aliphatic chain motions) match those found for different components in DS and MS relaxations. The conclusion is that secondary $\gamma$-relaxation processes are not due to thermally activated single molecular motions, but to a more complicated mechanism probably involving the concerted occurrence of several motions.

\section{ACKNOWLEDGMENTS}

We acknowledge "Donostia International Physics Center," and the funding support of the following research projects: Project No. MAT 2004/01017 and Project No. 9/UPV00206.215-13568/2001.

\section{APPENDIX A}

Neglecting the contribution of the single $H_{c}$ in PHd11, the quasielastic contribution can be approximated by

$$
I_{\text {qelas }}^{d 11}(Q, \omega) \simeq \frac{\sigma_{H a r}}{\sigma_{\text {tot }}^{d 11}} S_{H a r}^{q e l}(Q, \omega) .
$$

On the other hand, for the PHd6 sample we have

$$
I_{q e l a s}^{d 6}(Q, \omega) \simeq \frac{\sigma_{H a r}}{\sigma_{\text {tot }}^{d 6}} S_{H a r}^{q e l}(Q, \omega)+\frac{\sigma_{H c}}{\sigma_{\text {tot }}^{d 6}} S_{H c}^{q e l}(Q, \omega) .
$$

If, as shown in Fig. 7, it holds that

$$
I_{\text {qelas }}^{d 11}(Q, \omega) \frac{\sigma_{\text {tot }}^{d 11}}{\sigma_{\text {Har }}} \simeq I_{\text {qelas }}^{d 6}(Q, \omega) \frac{\sigma_{\text {tot }}^{d 6}}{\sigma_{\text {Har }}+\sigma_{H c}},
$$

by using the expressions (A1) and (A2) it follows that

$$
S_{H a r}^{q e l}(Q, \omega) \simeq S_{H c}^{q e l}(Q, \omega) .
$$

This implies that the quasielastic contribution for the localized motions involved, $S^{q e l}(Q, \omega)=(1-A) * \phi(\omega, \Gamma)$, are similar. It could happen that a balance between a very large displacement and a long characteristic time led to a similar quasielastic contribution rather than a small displacement and a shorter characteristic time. However, large displacements at very short times seem unrealistic and it is more reasonable to think that fast motions of $H_{a r}$ and $H_{c}$ will not considerably differ in amplitude. That is, their $(1-A)$ functions will be similar.

\section{APPENDIX B}

For two statistically independent motions $\alpha$ and $\beta$, 


$$
\begin{aligned}
I_{\text {qelas }}(Q, t)= & A_{\alpha}(Q)\left[1-A_{\beta}(Q)\right] e^{-t / \tau_{\beta}}+A_{\beta}(Q)[1 \\
& \left.-A_{\alpha}(Q)\right] e^{-t / \tau_{\alpha}}+\left[1-A_{\alpha}(Q)\right][1 \\
& \left.-A_{\beta}(Q)\right] e^{-t / \tau_{\beta}} e^{-t / \tau_{\alpha}} .
\end{aligned}
$$

When $\tau_{\alpha} \gg \tau_{\beta}$, then

$$
e^{-t / \tau_{\beta}} e^{-t / \tau_{\alpha}} \simeq e^{-t / \tau_{\beta}},
$$

and

$$
I_{\text {qelas }}(Q, t) \simeq\left[1-A_{\beta}(Q)\right] e^{-t / \tau_{\beta}}+A_{\beta}(Q)\left[1-A_{\alpha}(Q)\right] e^{-t / \tau_{\alpha}} .
$$

If the distribution of characteristic times originates from the packing heterogeneity of polymers then, for a $\mathrm{H}$ atom in a certain environment with a given packing degree, $\tau_{\alpha}$ and $\tau_{\beta}$ will be related so that low values for $\tau_{\alpha}$ will be accompanied by low values for $\tau_{\beta}$ and the other way around. As a consequence,

$$
\begin{aligned}
I_{\text {qelas }}(Q, t) \simeq & {\left[1-A_{\beta}(Q)\right] \sum f\left(\tau_{\beta}\right) e^{-t / \tau_{\beta}}+A_{\beta}(Q)[1} \\
& \left.-A_{\alpha}(Q)\right] \sum f\left(\tau_{\alpha}\right) e^{-t / \tau_{\alpha}} .
\end{aligned}
$$

As an illustration, Fig. 5 shows the $Q$ dependence for the case

$$
\sum f\left(\tau_{\text {flip }}\right) \frac{d\left(e^{\left.-t / \tau_{\text {flip }}\right)}\right.}{d \log _{10} t}=0.08 \sum f\left(\tau_{\text {short }}\right) \frac{d\left(e^{\left.-t / \tau_{\text {short }}\right)}\right.}{d \log _{10} t} .
$$

[1] N. G. McCrum, B. E. Read, and G. Williams, Anelastic and Dielectric Effects in Polymeric Solids (Dover, New York, 1967).

[2] F. Kremer and A. Schönhals, Broadband Dielectric Spectroscopy (Springer-Verlag, Heidelberg, 2002).

[3] M. Wehrle, G. P. Hellmann, and H. W. Spiess, Colloid Polym. Sci. 265, 815 (1987).

[4] A. K. Roy, A. A. Jones, and P. T. Inglefield, Macromolecules 19, 1356 (1986).

[5] J. J. Dumais et al., Macromolecules 19, 1884 (1986).

[6] M. T. Hansen, A. S. Kulik, K. O. Prins, and H. W. Spiess, Polymer 33, 2231 (1992).

[7] J. F. Shi, P. T. Inglefield, A. A. Jones, and M. D. Meadows, Macromolecules 29, 605 (1996).

[8] H. Kaji, T. Tai, and F. Horii, Macromolecules 34, 6318 (2001).

[9] A. Arbe, U. Buchenau, L. Willner, D. Richter, B. Farago, and J. Colmenero, Phys. Rev. Lett. 76, 1872 (1996).

[10] A. Arbe, D. Richter, J. Colmenero, and B. Farago, Phys. Rev. E 54, 3853 (1996).

[11] A. Arbe et al., Macromolecules 31, 4926 (1998).

[12] D. Richter et al., Macromolecules 31, 1133 (1998).

[13] S. Arrese-Igor et al., J. Chem. Phys. 120, 423 (2004).

[14] S. Arrese-Igor et al., J. Chem. Phys. 123, 14907 (2005).

[15] S. Arrese-Igor et al., J. Chem. Phys. 122, 049902(E) (2005).

[16] S. Arrese-Igor et al., J. Non-Cryst. Solids 352, 5072 (2006).

[17] I. Quintana, A. Arbe, J. Colmenero, and B. Frick, Macromolecules 38, 3999 (2005).

[18] A. Alegría, O. Mitxelena, and J. Colmenero, Macromolecules 39, 2691 (2006).

[19] S. W. Lovesey, Theory of Neutron Scattering from Condensed Matter (Oxford University Press, New York, 1987).

[20] F. Alvarez et al., Macromolecules 36, 238 (2003).

[21] A. C. Genix et al., Macromolecules 39, 3947 (2006).

[22] B. Frick, D. Richter, and C. Ritter, Europhys. Lett. 9, 557 (1989).

[23] D. Bedrov, G. D. Smith, and W. Paul, Phys. Rev. E 70, 11804 (2004).

[24] M. Bée, Quasielastic Neutron Scattering (Adam Hilger, Bristol, 1988).
[25] M. Wehrle, Doctoral Thesis, University of Mainz (1986).

[26] D. Fernandez, J. M. Alberdi, and J. Colmenero (private communication) (unpublished).

[27] P. M. Henrich and V. A. Nicely, Macromolecules 24, 2506 (1991).

[28] J. Bizerano and H. A. Clark, Macromolecules 21, 585 (1988).

[29] S. Arrese-Igor et al., Physica B 350, 971 (2004).

[30] B. Frick, C. Alba-Simionesco, J. Hendricks, and L. Willner, Prog. Theor. Phys. Suppl. 126, 213 (1997).

[31] B. Frick and C. Alba-Simionesco, Physica B 266, 13 (1999).

[32] B. Frick and C. Alba-Simionesco, Appl. Phys. A: Mater. Sci. Process. 74, s549 (2002).

[33] C. L. Soles et al., Macromolecules 34, 4082 (2001).

[34] TOSCA, TOF instrument at ISIS.

[35] J. W. Kang, K. Choi, W. H. Jo, and S. L. Hsu, Polymer 26, 7079 (1998).

[36] A. F. Yee and S. A. Smith, Macromolecules 14, 54 (1981).

[37] M. Hutnik, A. S. Argon, and A. W. Suter, Macromolecules 24, 5970 (1991).

[38] J. H. Dhih and C. L. Chen, Macromolecules 28, 4509 (1995).

[39] C. L. Chen, H. L. Chen, and J. H. Shih, Macromolecules 27, 2087 (1994).

[40] C. L. Chen, C. L. Lee, H. L. Chen, and J. H. Shih, Macromolecules 27, 7872 (1994).

[41] T. Shi, W. Jiang, L. An, and B. Li, Macromol. Theory Simul. 10, 232 (2001).

[42] C. F. Fan, T. Çagin, Z. M. Chen, and K. A. Smith, Macromolecules 27, 2383 (1994).

[43] C. F. Fan, T. Çagin, and W. Shi, Macromol. Theory Simul. 6, 83 (1997).

[44] S. F. Tsai, I. K. Lan, and C. L. Chen, Comput. Theor. Polym. Sci. 8, 283 (1998).

[45] A. Alegría, S. Arrese-Igor, and J. Colmenero, J. NonCrystalline Solids, 4th IDS/DRP Conference (2006) (to be published).

[46] S. Arrese-Igor, A. Alegría, B. Frick, and J. Colmenero (unpublished).

[47] M. Ochi, H. Kageyama, and M. Shimbo, Polymer 29, 320 (1988). 
[48] T. Takahama and P. Geil, J. Polym. Sci., Polym. Phys. Ed. 20, 1979 (1982).

[49] J. M. Margolis, Engineering Thermoplastics (Marcel Dekker, Inc., New York, 1985).

[50] S. Cerveny and A. Alegría (personal communication) (unpublished).

[51] D. Perchak, J. Skolnick, and R. Yaris, Macromolecules 20, 121 (1987)

[52] D. R. Whitney and R. Yaris, Macromolecules 30, 1741 (1997).

[53] C. A. Klung et al., Macromolecules 30, 6302 (1997).

[54] J. M. Goetz, J. Wu, A. F. Yee, and J. Schaefer, Macromolecules 31, 3016 (1998).

[55] J. Liu, A. F. Yee, J. M. Goetz, and J. Schaefer, Macromolecules 33, 6849 (2000).
[56] H. Kaji, F. Fuke, and F. Horii, Macromolecules 36, 4414 (2003)

[57] Fourier transforms were obtained by a noncommercial f77 program. This program basically (i) centers the maximum of the spectra in the energy window for slight deviations, (ii) corrects the experimental spectra from detailed balance factor, (iii) mirrors the experimental spectra for neutron energy losses experimentally not accessible, and (iv) performs a cosine discrete Fourier transform at time steps equal to $2 \pi / \omega_{\max }$ from $t_{\text {ini }}$ $=2 \pi / \omega_{\max }$ on, where $\omega_{\max }$ is the maximum energy transfer experimentally measured.

[58] NS mean characteristic times have been obtained from the mean activation energy values via the Arrhenius equation with $\tau_{\infty}=10^{-13} \mathrm{~s}$. 